This has led to some of the most important results in number theory.

While many of the proofs are clearly expounded, two criticisms might be made. The treatment is now and then condensed and calculations are sometimes omitted. It is also occasionally assumed that the reader is familiar with results which he reasonably might not know. Thus in the proof of the formula for $\pi_{2}(n)$, in Chapter 5, reference is made without explanation to "the method of stopping at an even term," and this really means that one assumes that

$$
1-k+\frac{k \cdot k-1}{2 !}+\cdots+\frac{k \cdot k-1 \cdots k-n+1}{n !}>0
$$

if $n$ is even. So in Chapter 12, on transcendental numbers, reference is made to a theorem of Polya without any statement of the theorem, which however is given in Vol. 2 of Pólya and Szegö's Aufgaben und Lehrsätze, Problem 75, Chapter V.

The other criticism refers to results in which formidable calculations play an important part. They are sometimes set out in such a manner that it is not easy to follow the sequence of operations, and to see how they are changing from line to line.

The details given in this review show that very large cross sections of results in number theory are contained in this book and that diverse methods and proofs are given. The chapters can be read independently of each other and so the reader may browse among them. $\mathrm{He}$ is sure to find much of interest to him whatever his tastes are. How fortunate he is in having this opportunity of becoming acquainted with so many exciting aspects of number theory, and this without preliminary study. The authors have done a great service to all interested in number theory, and readers will be very grateful to them.

\title{
L. J. MORDELL
}

Electrodynamics and classical theory of fields and particles. By A. O.

Barut. The Macmillan Company, New York, 1964.

Many mathematicians are not aware that recent work in theoretical physics has taken a strongly mathematical turn and has posed problems that would be of professional interest to a wide variety of mathematicians. This book is an excellent place to begin to sample this work: It is an exposition by a physicist of those parts of prequantum field theory that are most important for understanding quantum field theory, and has a clear, geometric-Lie group flavor that the reviewer finds very attractive. 
Chapter 1 deals with the geometrical space-time as a homogeneous space of the Lorentz group, and Chapter 2 with relativistic particle dynamics. It is very inspiring to see how neatly the mechanics falls out of the condition of covariance with respect to the Lorentz group. Although this was Minkowski's insight, it has only recently filtered down to the textbooks, and is still probably not known to most mathematicians, despite the fact that it is an ideal illustration of the sort of contribution a mathematician can make to physics.

Chapter 3 is "Relativistic field theory." The author proceeds from the examples of Maxwell's equations and formal passage to the limit from the mechanics of point particles to show how, using the formalism of the calculus of variations, the general idea of a "free field" that is covariantly related to Minkowski geometry can be formulated. We might remark that the use of the calculus of variations is not at all essential here, and is, from the mathematical point of view, a historical accident. The "right" framework is that of homogeneous vector bundles and their invariant differential operators. However, the variational formalism is handled very neatly here-although this is not true of most of the physics literature-and is certainly adequate for the immediate goals.

This completes Part I, which is called "Relativistic description of fields and particles"; Part II is called "Interactions of fields and particles." Now, the main difficulty in quantum field theory (and in the rest of theoretical physics today, for that matter) is in understanding how interactions can be formulated and calculated in a Lorentzinvariant framework. These problems also appear, in a simpler form, in pre-quantum physics, beginning with Lorentz' theory of the electron and carried further by such physicists as Fokker, Schwarzschild, Dirac, Wheeler and Feynman. This material that is collected together in the rest of the book is of the highest value to one trying to understand the analogous problems in quantum theory, as well as of great intrinsic interest. The basic mathematics is fascinating, involving non-linear (but usually only mildly non-linear) coupled ordinary and partial differential equations and the singularities of their solutions. One can only note in amazement that there seem to be very few contributions by mathematicians to its development.

Chapter 4 sets up the machinery (using the calculus of variations again) for writing down the coupled equations determining the interaction of fields and particles or two fields. Chapter 5, titled "Radiation and radiation reaction," is the climax of the book. The author puts his goals in the following way: "In this chapter we concentrate, in detail, on the electromagnetic radiation. First, the field of a mov- 
ing charged particle is discussed; then we define the radiation field and investigate its properties. This is followed by a discussion of the effect of the radiation field on the charged particle and the modified equations of the motion of the particle with radiation reaction which present unusual features. Additional requirements must be used to obtain physically meaningful solutions. Finally we discuss the classical mass renormalization and the problem of the electromagnetic nature of the mass of the electron. In the application of the results to the real physical situation, particularly when one speaks of the nature of the electron, quantum effects must be taken into effect. But the problems discussed here are fundamental for the consistency of the classical theory and what we may expect when the theory is quantized."

The last chapter, called "Action at a distance electrodynamics," discusses briefly an alternate approach to interactions, involving only "particles" via the "Fokker-Schwarzschild-Tetrode" action principle. The basic mathematics now is ordinary integro-differential equations, and one can only note again, wistfully, how much bolder and relevant the physicists have been than the mathematicians, and how useful would be solid existence and uniqueness theorems in clarifying the subject.

It is hard to say exactly what background a mathematician would need to read the book. The author recommends Classical electrodynamics by J. D. Jackson as a prerequisite; the reviewer has found Landau and Lifshitz' The Classical theory of fields very useful in filling in the gaps in his physics education. There are many comments, references and exercises scattered throughout that lead the reader into the literature.

The greatest obstacles to a mathematician grasping the problems that are on the frontiers of physics is his lack of conditioning in the fundamentals and his own overspecialized training and research. Ideally, we mathematicians should write our own expositions of basic physics, so as not to cut our profession off from its historical roots; until then, we can only applaud efforts such as this one that bridge the gap, and hope that more such striking expositions will be written.

Robert HermanN 